\title{
Situación sociodemográfica y nivel de satisfacción con la formación de los graduados de una universidad privada de la ciudad de Manizales, Colombia
}

\section{Socio-demographic situation and level of satisfaction with the training of graduates of a private university in the city of Manizales, Colombia}

\author{
Diana Lorena Cardona Montoya** \\ María del Pilar Cerezo Correa*** \\ Laura Patricia Giraldo Vélez **** \\ María Mercedes Naranjo Aristizábal ***** \\ María del Carmen Vergara Quintero ******
}

\begin{abstract}
**Ingeniera Mecánica. Especialista en Mantenimiento Industrial. Docente Instructor. Coordinadora Programa de Ingeniería Mecánica. Grupo de investigación de Diseño mecánico y desarrollo industrial "ARCHYTAS”. dcardona@ autonoma.edu.co. Antigua Estación del Ferrocarril. Manizales, Caldas, Colombia.

***Odontóloga, Especialista en salud en Investigación y Docencia Universitaria, Especialista en Auditoria en Salud, Magíster en Salud Pública. Docente Asociado. Grupo de investigación en Salud Pública. mapice@autonoma.edu. co. Antigua Estación del Ferrocarril. Manizales, Caldas, Colombia.

****Economista, Especialista en Desarrollo Gerencial, Magíster en Administración. Docente Asistente. Coordinadora del programa de Economía. Grupo de investigación Empresariado.pgiraldo@autonoma.edu.co. Antigua Estación del Ferrocarril. Manizales, Caldas, Colombia.

*****Licenciada en Educación, Fisioterapeuta, Magister en Neurorehabilitación. Docente asociado. Grupo de investigación Cuerpo-movimiento. mmnaranjo@autonoma. edu.co. Antigua Estación del Ferrocarril. Manizales, Caldas, Colombia.

******Odontóloga, Magister en Administración en salud Doctora en Ciencias Sociales. Niñez y Juventud. Docente Titular. Coordinadora de la Unidad de Investigación, Coordinadora e investigadora del proyecto de seguimiento a graduados UAM. Grupo de investigación en Salud pública. mcvq@autonoma.edu.co. Antigua Estación del Ferrocarril.

Manizales, Caldas, Colombia.
\end{abstract}

\section{Resumen}

Esta investigación tuvo como objetivo analizar la situación sociodemográfica, el nivel de satisfacción con las competencias y el nivel de identidad institucional de los graduados. La metodología tuvo alcance descriptivo, longitudinal retrospectivo. La población estuvo conformada por 2038 graduados de las facultades de salud, ingeniería y estudios sociales y empresariales entre 2008 y 2012 . Se utilizó un cuestionario de auto diligenciamiento al momento del grado. Se encontró que en la Facultad de Ingeniería la mayoría son hombres, en las tres facultades hay mayor proporción de solteros. El dominio de competencias relacionadas con el uso de la información agrupa el mayor porcentaje satisfecho en las tres facultades, con la competencia identificar y utilizar símbolos para comunicarse está satisfecho un menor porcentaje. En relación con los recursos físicos, el promedio más bajo de satisfacción se presenta con los espacios deportivos y para realizar actividades artísticas y el más alto con la biblioteca y aulas informáticas. Más del $79 \%$ está satisfecho con la formación académica, la fundamentación teórica y las relaciones interpersonales de los docentes, una menor proporción está satisfecho con el trabajo de campo. Más del $75 \%$ de los graduados de las tres facultades volvería a estudiar en la universidad, principalmente por la calidad de la formación. Finalmente los graduados tienen identidad con la institución, evidenciada en los porcentajes de satisfacción con el desarrollo de las competencias, los docentes, los recursos físicos y con algunos espacios físicos.

Palabras clave: Autoevaluación, calidad, graduados, pertinencia, seguimiento 


\begin{abstract}
This research aims at analyzing the socio-demographic situation, the level of satisfaction regarding the mastery of skills and the degree of institutional identity of graduates. The methodology had a descriptive, longitudinal and retrospective scope. 2038 graduates between 2008 and 2012 and who belonged to the health, engineering and social and business studies faculties participated in this research. They did a survey at the moment of their graduation. The results showed that most of the graduates from the engineering faculty were men, and there was a higher rate of singleness within the three faculties. The mastery of skills related to the satisfactory use of information gathered the highest percentage within the three faculties, whereas competences to identify and use symbols to communicate showed a lower percentage. In relation to facilities, the lowest satisfaction percentage was to sports and artistic locations, whereas the highest was for the library and computer rooms. More than $79 \%$ are satisfied with the academic training, theoretical foundations and interpersonal relationships with professors, while a smaller proportion are satisfied with fieldwork. Over $75 \%$ of graduates from the three faculties would come back to study at institution, mainly due to its quality in training. As a conclusion, graduates report to have identity with the institution. This is evident through the percentages of satisfaction with skills development, professors, physical resources and some facilities.
\end{abstract}

Keywords: Graduates, follow-up, quality, relevance, self-assessment.

\section{Introducción}

Esta investigación desarrollada en una institución de educación superior-IES- es un estudio de orden longitudinal descriptivo que se constituye en una estrategia de conocimiento de la realidad de los graduados, al tiempo que permite retroalimentar a los currículos y a la misma universidad como parte de su cultura de mejoramiento continuo de la calidad. Se realiza semestralmente mediante la recolección de la información con una encuesta auto diligenciada por los graduados en el momento del grado (M0), al año (M1), a los tres años (M3) y a los cinco años (M5) después del grado.

En la IES se han desarrollado cinco proyectos de seguimiento a graduados:

- Los dos primeros, fueron investigaciones desarrolladas en los programas de Odontología y Fisioterapia como parte del proceso de autoevaluación de los programas, los cuales permitieron caracterizar sus graduados socio-laboralmente y conocer sus perspectivas frente a los procesos de formación recibidas.

- El tercero consistió en la primera fase del proyecto de seguimiento a graduados, desarrollado en el año 2007-2008, financiado por el Ministerio de Educación Nacional-MEN; este permitió caracterizar la situación social, laboral, académica, familiar y la satisfacción con la formación recibida de los graduados de los programas de pregrado 2001-2008.
- El cuarto proyecto, lo constituyó el seguimiento a graduados segunda fase, apoyado por el MEN, cuyo producto final estuvo organizado en dos componentes: el primero permitió caracterizar la situación social, laboral, académica, familiar y la satisfacción con la formación recibida de los graduados de los programas de pregrado 2001-2008 y el segundo fue el diseño de una metodología para evaluar la calidad y la pertinencia de los programas académicos de pregrado en la que se articularon los graduados, los empleadores, el Proyecto Educativo del Programa -PEP- y el Proyecto Educativo Institucional -PEI-.

- Dando continuidad al proceso desarrollado por dos años consecutivos, durante el 2010, se realizó la tercera fase del proyecto de seguimiento a graduados, financiado igualmente por el MEN, proceso que validó la metodología en tres programas académicos de la IES, como parte de una prueba piloto, en la que se incluyó: Fisioterapia de la Facultad de Salud, Economía de la Facultad de Estudios Sociales y Empresariales e Ingeniería Electrónica de la Facultad de Ingeniería. En este se hicieron algunas modificaciones, como la incorporación de profesores en el proceso evaluativo y la introducción de grupos focales con graduados, empleadores y comités de currículo con el fin de cualificar los resultados obtenidos en las encuestas. 
En este artículo se presentan los resultados de la encuesta M0, es decir, en el momento del grado de los años 2008 al 2012, que permite caracterizar a los graduados en relación con las variables sociodemográficas, el desarrollo de las competencias, la satisfacción con los profesores, los recursos físicos y el nivel de identidad con la institución. Esta información es posible desagregarla por facultades y por programas y es utilizada particularmente por los comités de currículo como parte de su reflexión para la toma de decisiones en los procesos de autoevaluación con fines de mejoramiento continuo.

Los objetivos del estudio son:

- Identificar las características sociodemográficas de los graduados de los programas.

- Identificar el nivel de satisfacción de los graduados de pregrado con la formación recibida y los recursos ofrecidos por la institución.

- Analizar la percepción sobre el nivel de desarrollo de las competencias generales en el proceso de formación de los graduados.

- Conocer las perspectivas de desarrollo profesional de los graduados de la IES en el momento de graduarse.

Para responder a lo anterior, cada graduado de los programas de pregrado, durante los cinco años de estudio (2008 al 2012) diligenció la encuesta en el momento del grado. Dicha información fue sistematizada en cada periodo académico y analizada por cada programa académico y por las tres facultades de la institución. En este artículo se presenta la información por facultades.

El seguimiento a los graduados en la institución, se ha constituido en un proceso de evaluación continua y mejoramiento de la calidad, y en una investigación permanente que permea el desarrollo curricular a nivel de las facultades y los programas académicos, reconociendo a sus graduados como actores claves en la dinámica institucional, dado que son las personas que desde su experiencia personal y profesional pueden retroalimentar los currículos de los programas, en respuesta a las demandas del trabajo en los campos de actuación profesional específicos y en coherencia con las necesidades de la región.
Por lo anterior, el seguimiento a los graduados se constituye en un eje de trabajo del direccionamiento estratégico, que responde a las políticas de orden nacional e institucional en el marco de la cultura de los procesos de autoevaluación y mejoramiento continuo de la calidad de los programas tanto de pregrado como de posgrado. Siguiendo los planteamientos del Consejo Nacional de Acreditación, la cultura de la evaluación es una estrategia que posibilita el mejoramiento continuo de la calidad. Una de las tendencias mundiales más significativas del sector educativo de hoy se centra en la calidad (Maya \& Herrera, 2012). Bajo este propósito de calidad, las instituciones de educación superior, han promovido diversas estrategias dentro de las que se reportan el proceso de seguimiento a graduados como una posibilidad de analizar la pertinencia de la formación y el impacto de los mismos en la sociedad y en el mundo laboral.

La calidad y pertinencia en la educación superior se constituyen en aspectos que si bien son diferentes deben analizarse en conjunto, como factores que se autodeterminan mutuamente, de hecho tanto la calidad como la pertinencia funcionan en doble vía, de la universidad hacia la sociedad y de esta a la universidad. En este sentido no es posible lograr una educación pertinente con procesos de formación de baja calidad, de allí que ambos procesos son factores que deben hacer parte de la agenda de las IES desde el enfoque de la responsabilidad social y de los procesos de autoevaluación y mejoramiento continuo de la calidad.

Los retos que impone una educación de calidad están relacionados con la capacidad de la formación para cumplir con las expectativas del graduado de los niveles técnico, tecnológico, profesional o de posgrados, las cuales están referidas al desarrollo intelectual, mayor estatus, movilidad social y mejores ingresos; es decir, una formación que permita mejorar el desempeño laboral a través del desarrollo de competencias requeridas por las organizaciones y las empresas, de tal manera que puedan ser capaces de efectuar un aporte efectivo a la sociedad, contribuyendo a su desarrollo y crecimiento económico y social.

Lo anterior refleja la pertinencia de una institución formadora, la cual se constituye desde el eje de 
relación entre la universidad y el entorno. Es un factor determinante que tiene en cuenta la concordancia entre las misiones de las IES y las expectativas de la sociedad. La Conferencia Mundial sobre Educación Superior -Unesco- celebrada en París en 1998, definió que la pertinencia de la educación superior debe evaluarse en función de la adecuación entre lo que la sociedad espera de las instituciones y lo que estas hacen. Lo anterior, tal como se presenta en dicha conferencia, requiere que los países adopten una mirada ética y responsable de la educación, y se reten a responder a una mejor articulación con los problemas de la sociedad y el mundo del trabajo. En este escenario, parece imprescindible que cada institución de educación, analice y evalúe la calidad y pertinencia de la formación que están ofreciendo, esto es, reflexionen sobre el cumplimiento de su razón de ser y el desempeño de sus funciones, que les permitan mejores posibilidades para competir en el escenario mundial.

De esta manera, la responsabilidad de las instituciones educativas no concluye con el otorgamiento del título profesional, es necesario que estas realicen todos los esfuerzos posibles por mantener contacto con sus graduados, conocer sus condiciones en el mercado laboral y sus expectativas de formación continua (Mejía, Nieto, Arboleda, \& Montoya, 2012). Estos estudios han tomado fuerza por su propósito de contribuir a mejorar la calidad de la educación. El Ministerio de Educación colombiano dice:

Hacer seguimiento a los egresados de la educación superior es una tendencia creciente en países que buscan mejorar la calidad y la pertinencia de los programas académicos...ya que suministran insumos que las instituciones, el sector productivo, el gobierno y los estudiantes están usando para tomar decisiones. (Aldana de Becerra, Morales González, Aldana Reyes, Sabogal Camargo, \& Ospina Alfonso, 2008).

El seguimiento a los graduados se constituye entonces en un mecanismo para determinar la pertinencia de la formación recibida, así como la calidad de la misma, en tanto que permite determinar la coherencia entre la formación y las necesidades del mercado laboral y de una u otra manera permite evaluar el logro de los fines que una institución se ha propuesto cumplir en un determinado momento histórico. En este contexto, el egresado es una fuente importante de retroalimentación, en tanto que permite a la universidad conocer dónde y cómo está ubicado, su rol social y económico y la forma de reflejar los valores adquiridos durante su formación académica, aspectos que dan cuenta de la pertinencia de los programas y currículos de las Instituciones de Educación Superior (Aldana de Becerra, G. M., Morales González, F. A., Aldana Reyes, J. E., Sabogal Camargo, F. J., \& Ospina Alfonso, Á. R. 2008).

Bajo esta perspectiva, el seguimiento a los graduados se constituye en un eje para la reflexión curricular, en tanto que ofrece una línea de diagnóstico de la realidad que le permite a las instituciones reflexionar alrededor del análisis y la evaluación curricular, a partir de la valoración en retrospectiva que hace el graduado sobre la calidad de la formación recibida (Montenegro, 2011).

Según las teorías del capital humano, el egresado ha pasado de ser el profesional con conocimientos técnicos, a ser una persona en medio de intereses sociales en tanto es un ser social. Por tanto, tendrá que responder por el desempeño de sus tareas profesionales y al reconocerse como integrante activo de una sociedad - por sus intereses políticos, sociales o de estatus, haciéndolos coherentes con decisiones de política y de desarrollo (Ascun \& Red Seis, 2006).

El seguimiento a egresados ha de evaluar, dentro de un período determinado y de acuerdo con unos parámetros preestablecidos, la eficiencia profesional en función de la formación recibida, la aceptación en el mercado laboral y la correspondencia entre las áreas de especialización y las necesidades del país y ha de dar cuenta del cumplimiento de las funciones de una institución educativa; es decir, determinar en qué medida se están alcanzando los fines de la educación y si los objetivos institucionales y curriculares se cumplen (Aldana de Becerra, G. M., Morales González, F. A., Aldana Reyes, J. E., Sabogal Camargo, F. J., \& Ospina Alfonso, Á. R. 2008). La participación de los graduados en la vida institucional se constituye en una pieza clave para la conducción misional de la institución (Ascun \& Red Seis, 2006). En este sentido, debe hacer parte de la cultura institucional, un protagonista de la vida 
democrática del quehacer institucional en desarrollo de sus fines misionales (Ascun \& Red Seis, 2006).

\section{Materiales y métodos}

La investigación corresponde a un estudio cuantitativo longitudinal retrospectivo de alcance descriptivo; cuya población estuvo constituida por los graduados de todos los programas de pregrado en las tres facultades de salud (fs), ingeniería (fi) y estudios sociales y empresariales (fesye) entre los años 2008-2012 (2038 graduados). La muestra estuvo constituida por el $79,14 \%$ del total de la población de los graduados de la ies. La técnica de recolección de información utilizada fue la encuesta, y como instrumento se utilizó un cuestionario de auto diligenciamiento que se aplicó al momento del grado cada semestre. La encuesta contempla 7 apartados: información personal y familiar, historia académica y de financiación, desarrollo de competencias, plan de vida, situación laboral, nivel de identidad con la institución y satisfacción con los recursos ofrecidos; todos estos datos fundamentales para el seguimiento del graduado, en coherencia con lo planteado por el observatorio laboral de la educación. Para el proceso de sistematización de la información se diseñó la base de datos en spss versión 19, a partir de cual se realizó un análisis descriptivo de cada una de las variables, y se desarrolló un sistema de análisis bivariado y multivariado.

\section{Resultados}

A continuación se presentan los resultados del comportamiento de algunas variables entre las tres facultades, principalmente en las variables que aportan a la reflexión curricular y a la retroalimentación institucional, tales como la caracterización sociodemográfica, el nivel de satisfacción con el desarrollo de las competencias, así como el nivel de identidad y satisfacción con la institución.

Como se muestra en la tabla 1, participaron 1613 graduados entre el año 2008 a 2012, con un 79\% de representatividad frente al $100 \%$ de graduados de las tres facultades. La facultad con mayor porcentaje de seguimiento fue la facultad de salud con un $88 \%$.
Tabla 1. Porcentaje de seguimiento de los graduados por facultad entre 2008 y 2012

\begin{tabular}{lccc}
\hline Facultad & $\begin{array}{c}\text { Número de } \\
\text { graduados }\end{array}$ & $\begin{array}{c}\text { Número de graduados } \\
\text { que diligenciaron la } \\
\text { encuesta al momento } \\
\text { del grado }\end{array}$ & \% de seguimiento \\
\hline Salud & 681 & 601 & $88 \%$ \\
Ingeniería & 602 & 436 & $72 \%$ \\
Estudios sociales y & 755 & 576 & $76 \%$ \\
empresariales & 2038 & 1613 & $79 \%$ \\
Total & & & \\
\hline
\end{tabular}

Fuente: Registro Académico y base de datos del proyecto

\section{Características sociodemográficas}

Las características sociodemográficas de importancia para el presente artículo son el sexo y el estado civil de los graduados en el momento del grado, la ocupación de los padres y su respectivo nivel de formación, las cuales se comportaron de la siguiente manera en las tres facultades:

- En la FESYE y en la FS la mayoría son mujeres (FESYE 68\%, FS 71\%, FI 31\%) en relación con el estado civil, el mayor porcentaje en las tres facultades es soltero, (FESYE $71 \%$ son solteros y $21 \%$ casados, FS $86 \%$ son solteros y $12 \%$ casados y en FI el $85 \%$ son solteros y $9 \%$ casados). Los graduados que tienen hijos en su mayoría refieren tener un solo hijo.

- Con respecto a la educación de los padres se identificó que en la FESYE el 17\% cursó hasta la secundaria completa, $15 \%$ universitario completo, y el $12 \%$ posgrado y el nivel formativo de las madres es de secundaria completa $20 \%$, universitaria completa $14 \%$ y posgrado $11 \%$. Los padres en un $27 \%$ son trabajadores independientes y el $26 \%$ son empleados de empresas particulares y las madres el $41 \%$ se dedican a oficios del hogar, y 19\% son trabajadoras independientes.

- En la FS el nivel formativo para los padres es del $17 \%$ secundaria completa, educación universitaria completa $17 \%$ y educación de posgrado el $13 \%$ y la formación de la madres es secundaria completa $22 \%$, universitaria completa $14 \%$ y posgrado $18 \%$. La ocupación del padre es $31 \%$ trabajador independiente, $22 \%$ empelado del gobierno y las madres el $27 \%$ son empleadas del gobierno y el $26 \%$ oficios del hogar. 
- En la FI el nivel formativo para los padres es del $13 \%$ secundaria completa, educación universitaria completa $19 \%$ y educación de posgrado el $14 \%$ y la formación de la madres es secundaria completa $20 \%$, universitaria completa $17 \%$ y posgrado $14 \%$. La ocupación de los padres es de $31 \%$ trabajador independiente y el $25 \%$ empleado del gobierno y la ocupación de la madre es del $30 \%$ oficios del hogar y $22 \%$ empleado del gobierno.

\section{Satisfacción con el nivel de desarrollo de las competencias}

Las competencias se agruparon por áreas relacionadas con: el uso de la tecnología, la comunicación oral y escrita, el manejo de la información y las actitudes frente al trabajo.

Con respecto al uso de la tecnología se evaluaron la capacidad para utilizar herramientas informáticas básicas, crear, investigar y adoptar tecnología y diseñar e implementar soluciones con el apoyo de la tecnología. En la figura 1 se observa que en esta área un porcentaje superior al $85 \%$ de los graduados está muy satisfecho. La utilización de herramientas informáticas básicas es la de mejor calificación en las FESYE y FI.

Figura 1. Satisfacción de los graduados de las tres facultades con el desarrollo de las competencias relacionadas con el uso de la tecnología

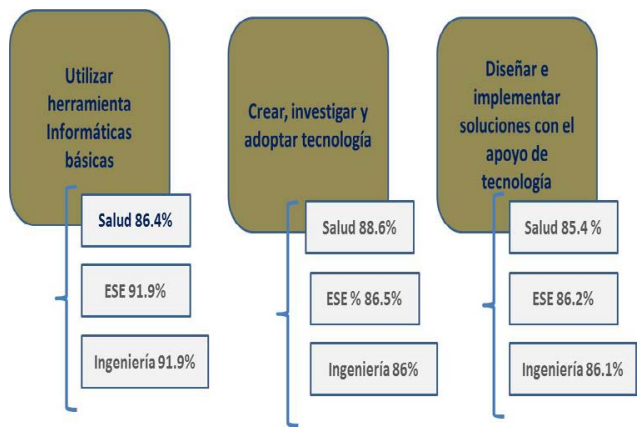

Fuente: elaboración propia

Las competencias relacionadas con la comunicación oral y escrita son la capacidad para exponer las ideas por medios escritos, comunicarse oralmente con claridad, lograr persuadir a los interlocutores e identificar y utilizar símbolos para comunicarse. Frente a esta área, tal como se muestra en la figura 2, la competencia de utilizar símbolos para comunicarse es la que menor satisfacción genera entre los graduados de las tres facultades.

Figura 2. Satisfacción de los graduados de las tres facultades con el desarrollo de las competencias relacionadas con la comunicación oral y escrita

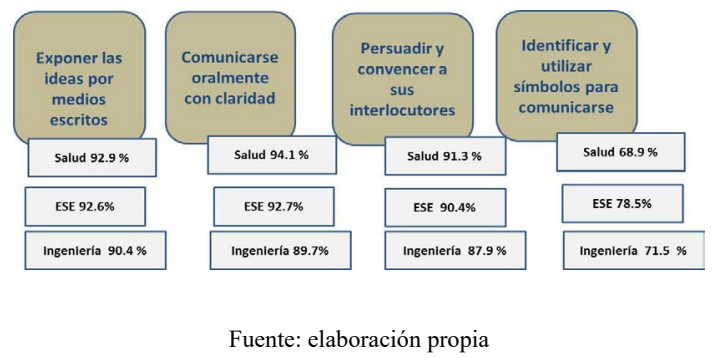

Frente al manejo de la información se incluyeron las competencias de buscar, analizar, administrar y compartir la información y la capacidad de abstracción, análisis y síntesis. El comportamiento del nivel de satisfacción con estas competencias se muestra en la figura 3 , en la que se hace evidente que por encima del $92 \%$ de los graduados de las tres facultades están satisfechos con el nivel de desarrollo de estas competencias; cerca del 96\% de los participantes de la Facultad de Salud están satisfechos con la capacidad de administrar, analizar y compartir información y por encima del $94 \%$ en la facultad de ESYE, con la capacidad de abstracción, análisis y síntesis.

Figura 3. Satisfacción de los graduados de las tres facultades con el desarrollo de las competencias relacionadas con el manejo de la información

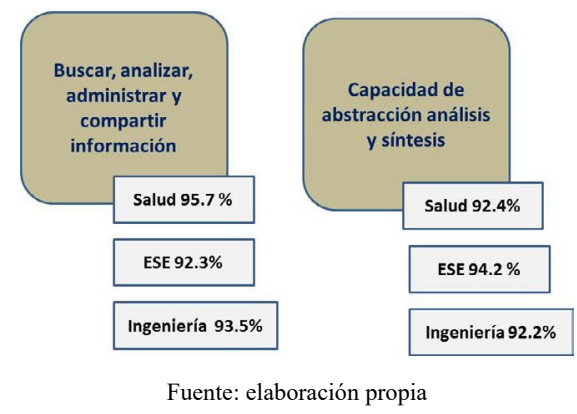


En el área de actitudes frente al trabajo se indagó sobre el nivel de satisfacción con las competencias de ser creativo e innovador, plantear y solucionar problemas, asumir responsabilidades, tomar decisiones, cultura de la convivencia, aceptar las diferencias y comprender la realidad que lo rodea. Frente al nivel de satisfacción con estas competencias, se evidencia en la figura 4 que por encima del $92 \%$ en las tres facultades está muy satisfecho con estas competencias. Nótese que el mayor porcentaje de satisfacción lo tiene la competencia de asumir responsabilidades y tomar decisiones, en más del $97 \%$ de los graduados y la que reporta una menor satisfacción es la relacionada con la creatividad e innovación.

Figura 4. Satisfacción de los graduados de las tres facultades con el desarrollo de las competencias relacionadas con las actitudes frente al trabajo
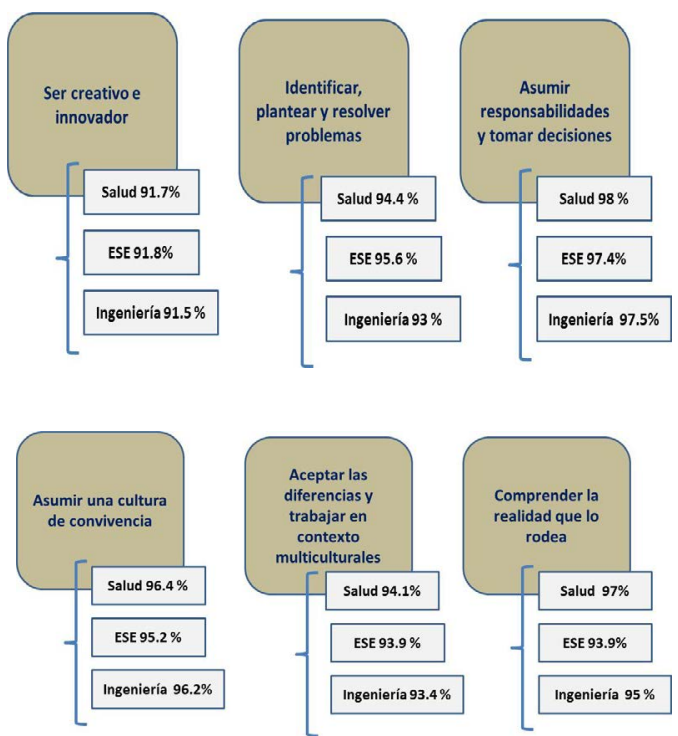

Fuente: elaboración propia

\section{Satisfacción con los recursos ofrecidos por la IES}

En este aspecto se indagó sobre el nivel de satisfacción de los graduados con: los docentes, el apoyo a los estudiantes, los recursos físicos y la gestión administrativa.
Con relación a los docentes se preguntó acerca de características relacionadas con la fundamentación conceptual, las relaciones interpersonales, la disponibilidad de tiempo y la formación académica. Encontrándose como se evidencia en la figura 5, los siguientes hallazgos:

Figura 5. Proporción de graduados de las tres facultades satisfechos con las características de los docentes

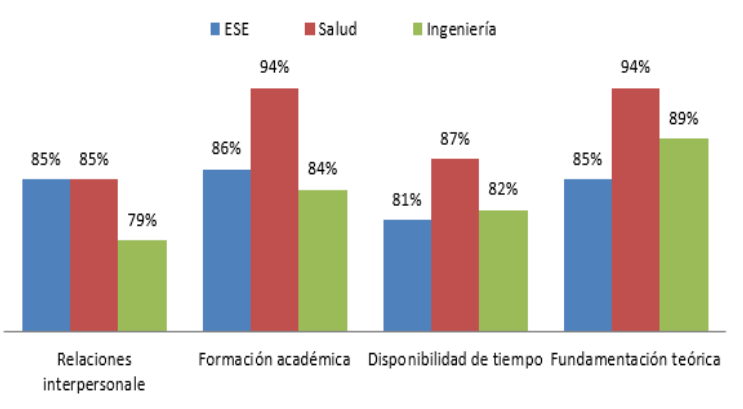

Fuente: elaboración propia

Más del $80 \%$ de los graduados de las tres facultades está satisfecho con las características de los docentes. Valores superiores al $84 \%$ de los graduados en las tres facultades reconoce en sus docentes la formación académica y la fundamentación teórica, con un porcentaje mayor del $94 \%$ en los graduados de la facultad de salud. Un $90 \%$ de los graduados de la Facultad de Salud está satisfecho con las cuatro características de los docentes, y de manera similar un $84 \%$ de los de la Facultad de Ingeniería y ESYE.

Con relación la satisfacción con el apoyo a los estudiantes se incluyeron aspectos relacionados con los procesos de aprendizaje, trabajo de campo, intercambios, prácticas, investigación, entre otras. En la figura 6, se muestra que el apoyo en procesos de aprendizaje es con el que mayor proporción está satisfecho y el apoyo en la gestión para identificar empleo es con el que menor proporción lo está en las tres facultades. Los graduados más satisfechos con todos los recursos son los de la Facultad de Salud. 
Figura 6. Proporción de graduados de las tres facultades satisfechos con los apoyos ofrecidos por la IES

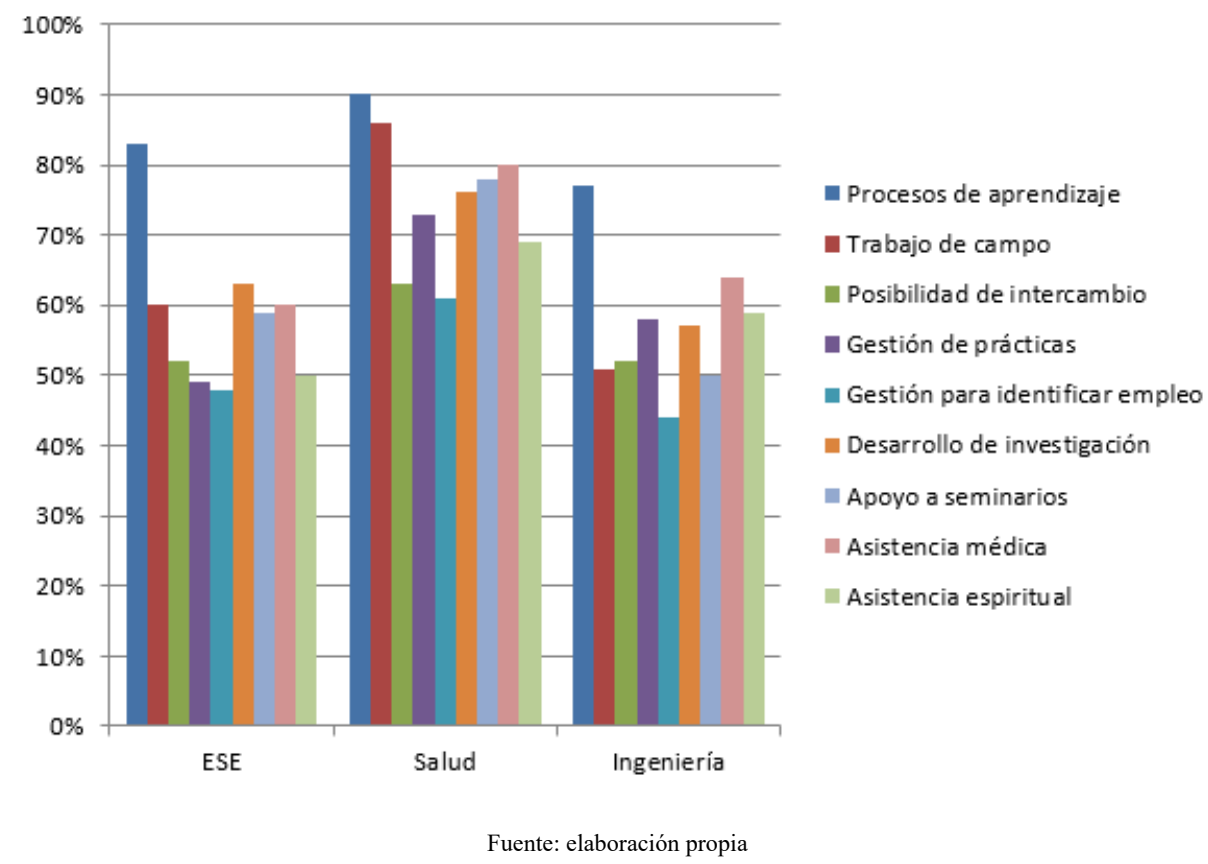

En los recursos físicos se incluyeron los espacios requeridos para la formación integral de los estudiantes. El comportamiento de los graduados satisfechos en las tres facultades es similar, principalmente en la satisfacción con la biblioteca (superior al 75\%) y con las aulas informáticas (superior al 67\%). La menor proporción de graduados satisfechos la tiene los espacios deportivos y para arte, con menos de un $60 \%$. Se evidencia que los graduados de la Facultad de Salud son, en términos generales, los más satisfechos con los recursos físicos (figura 7).

Figura 7. Proporción de graduados de las tres facultades satisfechos con los recursos físicos de la IES

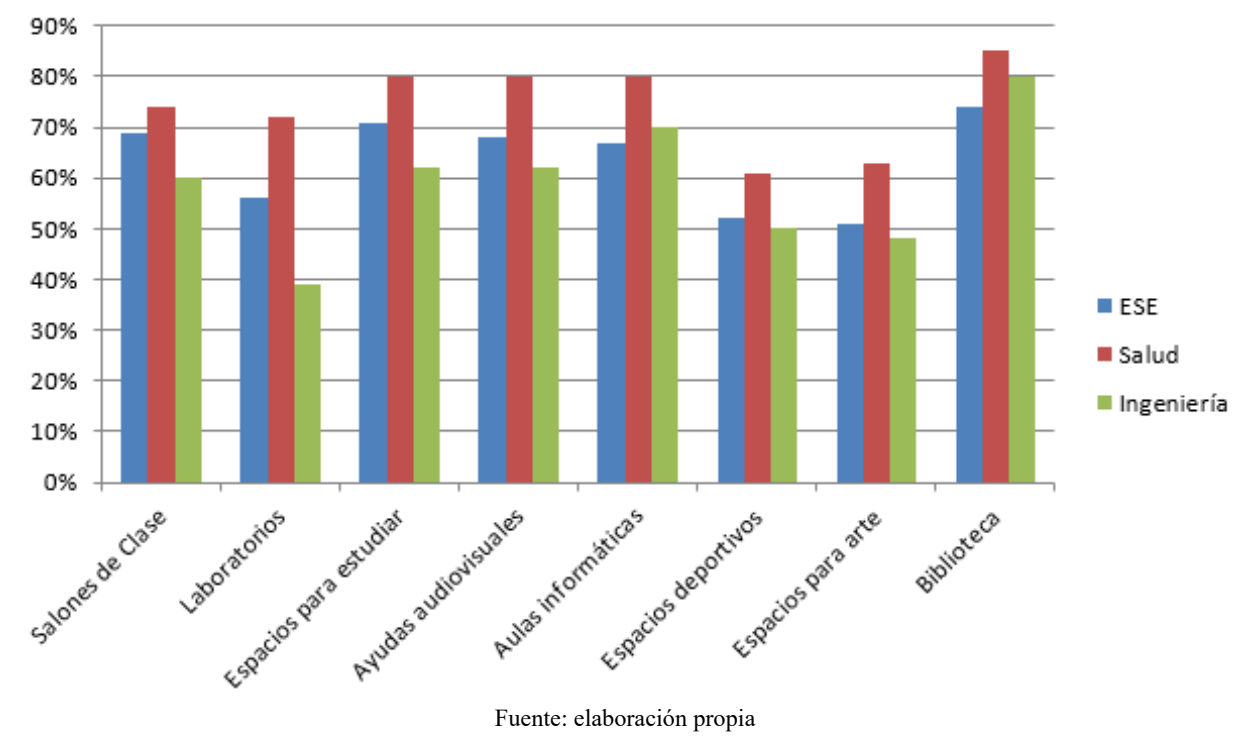


Con relación a la satisfacción con la gestión administrativa se evidencia en la figura 8 que cerca del $80 \%$ de los graduados de las tres facultades está satisfecho con la atención al personal, y en un porcentaje menor (por debajo del 70\%) con la agilidad en los trámites.

Figura 8. Proporción de graduados de las tres facultades satisfechos con los recursos físicos de la institución

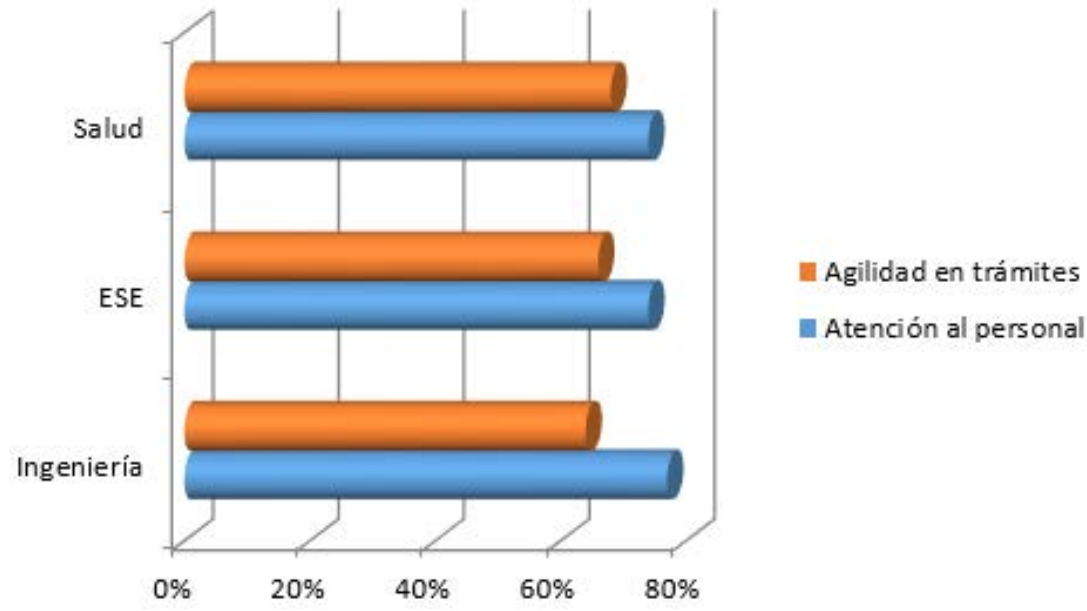

Fuente: elaboración propia

\section{Nivel de identidad de los graduados con la IES}

El nivel de identidad con la institución se indagó desde preguntas relacionadas con la recomendación que harían los graduados a otros bachilleres, la opción de volver a la institución y las razones para regresar o no.

Con relación a la opción de recomendación de la universidad a otros bachilleres, se encontró que entre el 75\% y el $85 \%$ de los graduados de las tres facultades sí recomendarían a la IES, principalmente con casi un $85 \%$ los graduados de la Facultad de Salud, como se muestra en la figura 9.

Figura 9. Proporción de graduados de las tres facultades que recomendaría a la IES

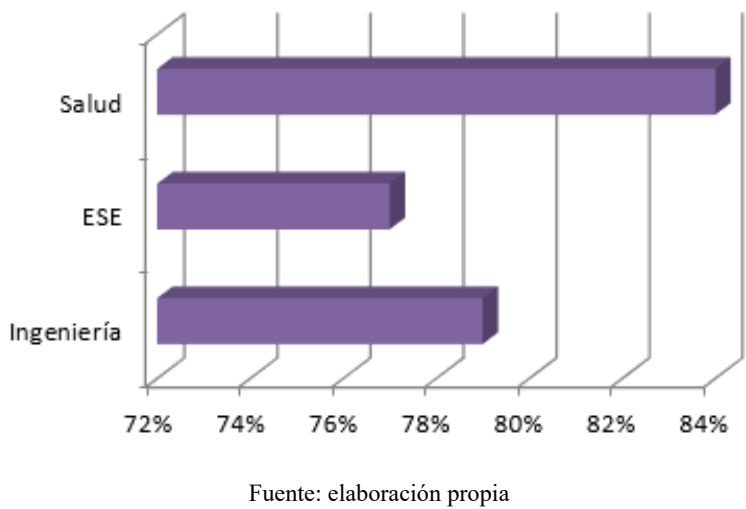


A la pregunta, si volvería a estudiar en la IES, se evidencia en la figura 10, que porcentajes superiores al $67 \%$ de los graduados de las tres facultades si volverían.

Figura 10. Proporción de graduados de las tres facultades que volverían a estudiar en la IES
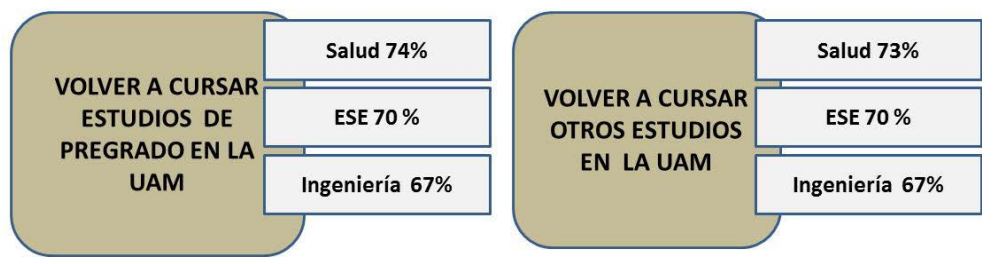

Fuente: elaboración propia

De los graduados que volverían, principalmente lo harían para realizar estudios universitarios, seguido de especializaciones y en menor proporción a realizar estudios de maestría, como se muestra en la tabla 2.

Tabla 2. Tipo de estudios que estudiarían los graduados en la IES

\begin{tabular}{lc}
\hline Tipo de estudios & Porcentaje \\
\hline Universitarios & $32 \%$ \\
Especializaciones & $33 \%$ \\
Maestría & $9 \%$ \\
Diplomados & $16 \%$ \\
\hline \multicolumn{2}{c}{ Fuente: elaboración propia }
\end{tabular}

Con relación a las razones de los graduados para volver a la universidad, se encuentra que más del $41 \%$ de los graduados de las tres facultades volverían a la institución por la calidad en la formación, y solo un 1\% lo harían por las posibilidades de encontrar empleo, como se muestra en la tabla 3. De manera representativa entre el 15\% y el $20 \%$ volverían por el reconocimiento de la institución.

Tabla 3. Razones para volver a la IES

\begin{tabular}{|c|c|c|c|}
\hline Razones para volver & Salud & $\begin{array}{c}\text { Estudios sociales y } \\
\text { empresariales }\end{array}$ & Ingeniería \\
\hline Calidad de la formación & $56 \%$ & $41 \%$ & $42 \%$ \\
\hline Calidad de los profesores & $12 \%$ & $11 \%$ & $16 \%$ \\
\hline Reconocimiento de la institución & $17 \%$ & $20 \%$ & $15 \%$ \\
\hline Posibilidades de encontrar empleo rápidamente & $1 \%$ & $1 \%$ & $1 \%$ \\
\hline Fundamentación para crear empresa & $1 \%$ & $11 \%$ & $3 \%$ \\
\hline Los recursos de apoyo a la formación & $1 \%$ & $9 \%$ & $4 \%$ \\
\hline Otras & $1 \%$ & $2 \%$ & $5 \%$ \\
\hline No responde & $11 \%$ & $9 \%$ & $15 \%$ \\
\hline
\end{tabular}

Fuente: elaboración propia 


\section{Discusión de resultados}

Según el observatorio laboral de la educación, durante los años 2001 y 2012 se graduaron de profesiones tecnológicas y universitarias, de universidades públicas y privadas en el departamento de Caldas 5.577 personas de profesiones afines con las áreas de ciencias de la salud, 10.195 de las áreas de Economía, administración, contaduría y afines y 13.486 en las áreas de ingeniería, arquitectura, urbanismo y afines (OLE- Observatorio Laboral de la Educación, 2015). La Universidad Autónoma de Manizales, aporta a esta cifra en el periodo 2008 a 2012, 681 en el área de la salud, 755 en el área de economía, administración y afines, y 602 en el área de ingeniería.

En los tres programas de la Facultad de Salud se observó que la mayoría de los graduados son del sexo femenino, este resultado no se distancia de lo que ocurre a nivel nacional, por ejemplo, el Observatorio Laboral de la Educación reporta que el 56\% de los graduados entre los años 2001 y 2012 fueron mujeres; de manera similar ocurre en la Facultad de Estudios Sociales y Empresariales en donde la mayoría de los graduados son del sexo femenino. En contraste para la Facultad de Ingeniería la mayoría fueron hombres, esto se relaciona con lo que sucede a nivel nacional, en donde las instituciones buscan que las mujeres se interesen por el estudio de las ciencias e ingeniería, debido al desbalance en términos del género, en la toma de decisión de la carrera (UNAL, 2012).

Una investigación realizada en México mostró una creciente participación de las mujeres en la educación superior, evidenciada con el incremento constante en el número de mujeres matriculadas entre 1980 y 2005, periodo durante el cual prácticamente se duplicó. En 1980 se observaba la presencia de 54 mujeres por cada 100 hombres. Durante los quince años siguientes, los valores, aunque en aumento, se mantuvieron por debajo de 100 y a partir de 1995 superó el umbral de 100, lo que significa un proceso constante de feminización. A pesar de lo anterior, entre 1995 y 2005 se observó que en la carrera de odontología disminuyó la proporción de mujeres (Corleto, Cooper, Loredo, \& Bolleto, 2006).

Estudio recientes en el ámbito colombiano con los graduados de la Universidad Javeriana del año 2012 mostró que el 58\% de los graduados son del género femenino, es de resaltar que este estudio no hace referencia a una profesión en particular. Esta participación de las mujeres en los programas de educación superior, se podría traducir en mayor inclusión en los diferentes sectores productivos y promoción de la equidad social (MEN, 2012).

En Colombia se realizó un estudio para identificar los determinantes de la demanda en la educación superior, considerando las políticas de oferta educativa, préstamos y subsidios para la financiación en universidades privadas, encontrando que las mujeres tienen una mayor probabilidad de ingreso a la educación superior, caso contrario ocurre con las personas casada, divorciadas o separadas (Acevedo \& Gómez, 2015).

Considerando el estado civil, este estudio mostró que la mayoría de los graduados al momento del grado se encontraban solteros y sin hijos. Esta característica también se observa en otros estudios de graduados en Colombia como el de la Universidad Javeriana (Javeriana, 2012), en donde el 79,8\% eran solteros, el de la Fundación Universitaria del Área Andina, para el año 2011, con el $77.9 \%$ en el mismo estado civil (Rodriguez et al., 2012). Con excepción de las tecnologías de la IES, en el cual el porcentaje de graduados casados es mayor que en los otros programas, evidenciándose una fuerte relación entre la edad de grado de estas personas y la necesidad de formar una familia en una edad mediana de la vida, quienes en su mayoría son personas adultas, que trabajan y estudian simultáneamente.

Con relación al nivel educativo del padre de los graduados, se identificó que un porcentaje superior al $40 \%$ tiene estudios profesionales, con excepción de los padres de los graduados de algunas tecnologías. Estudios similares muestran porcentajes inferiores (Rodriguez et al., 2012) y (Javeriana, 2012). En cuanto al nivel educativo de las madres de los graduados, se identificó que un porcentaje superior al $50 \%$ tienen estudios profesionales, con excepción de las madres de los graduados de las tecnologías, para quienes los porcentajes son inferiores al $40 \%$. En estudios que pretenden establecer la relación entre el nivel educativo del padre y el acceso de sus hijos a la educación superior, se ha encontrado: "La educación de los padres es esencial en el logro escolar de los 
hijos" (Marí Klose, Marí Klose, Granados, Granell, \& Martnez, 2009, (p. 297)), en el mismo estudio se establece que el nivel de estudios de la madre es, el factor que más influye en el devenir educativo de los hijos.

Con porcentajes superiores al $50 \%$, los padres de los graduados del Programa de Economía refieren tener una posición ocupacional patrón o empleador, evidenciándose una relación en la influencia de la familia en la toma de decisiones de la carrera por parte de los graduados de la Facultad de Estudios Sociales y Empresariales, en contraste para los graduados de las facultades de salud e ingeniería, dicho porcentaje no supera el $20 \%$, prevaleciendo en estos casos la posición ocupacional trabajador independiente $\mathrm{o}$ empleado. En relación con la posición ocupacional de las madres, porcentajes inferiores al $20 \%$ son patronas o empleadoras, siendo 10 puntos más alto en la Facultad de Estudios Sociales y Empresariales, en su mayoría la posición ocupacional de las madres de los graduados es oficios del hogar, empleado del gobierno y patrón o empleador.

Otros estudios de seguimiento a graduados a nivel nacional, encuentran que la mayoría de los padres de los graduados son trabajadores independientes o empleados y porcentajes más bajos por cuenta propia y que las madres en su mayoría se dedican a labores del hogar (Brunal \& Martínez, 2008). Así mismo, ha encontrado que estudiantes con padres que se desempeñan en cargos superiores, como jefes o directivos, tienen mayor probabilidad de permanecer y de graduarse, comparado con aquellos alumnos cuyos padres se desempeñan como obreros o empleados sin mando(UAO- Oficina de Planeación y desarrollo Institucional, 2007).

Con respecto al rango de edad, la mayor parte de los estudiantes universitarios en Colombia tienen edades inferiores a los 23 años, en EAFIT, (Jaramillo \& Ruiz, 2001) la mayoría son menores de 23 años, en la Universidad Nacional sede Bogotá, el $33.08 \%$ son menores de edad, en la sede Manizales el 22.81\% tiene menos de 18 años, en la Universidad Javeriana - Cali, la mayoría de los estudiantes están en el rango de 17-20 años, el estudiante universitario de Bogotá se ubica entre los 18-24 años de edad. En la Universidad Autónoma de Manizales, la mayor parte de la población estudiantil se ubica entre los 17 y 25 años (88\%). En la Universidad de La Plata - Argentina, el $64 \%$ de los estudiantes tienen entre 21-23 años de edad (UAO- Oficina de Planeación y desarrollo Institucional, 2007).

Con relación a las competencias, los resultados de las investigaciones de seguimiento a los graduados en el momento del grado, permiten conocer como los nuevos profesionales perciben el desarrollo de las competencias que desarrollaron durante su formación y que son necesarias para enfrentarse al mundo laboral. Los graduados de las tres facultades durante el periodo de estudio, califican en un más alto porcentaje como muy satisfechos y satisfechos las competencias relacionadas con asumir responsabilidades y tomar decisiones, comprender la realidad que lo rodea, asumir una cultura de convivencia y aprender y mantenerse actualizado. Las competencias con las cuales un mayor porcentaje se siente insatisfecho o muy insatisfecho son identificar y utilizar símbolos para comunicarse, diseñar e implementar soluciones con el apoyo de la tecnología, utilizar herramientas informáticas básicas, crear, investigar y adoptar tecnología.

De forma similar el OLE en uno de sus informes muestra (MEN, 2012), que los recién graduados encuestados de las instituciones de educación superior del país, señalan que las competencias más fuertes son trabajo en equipo para alcanzar metas comunes y aplicar valores y ética profesional en el desempeño laboral, seguido por formular y ejecutar proyectos e identificar, plantear y resolver problemas. Por el contrario, las competencias más débiles son las asociadas con la utilización de herramientas informáticas especializadas, el trabajo bajo presión, la identificación y utilización de símbolos para comunicarse y la creación, investigación y adaptación de tecnología.

La explicación a la calificación que dan los egresados podría encontrarse en los resultados del proyecto Réflex (Aneca, 2008) que señala: Como consecuencia de la aplicación en el aula de metodologías de enseñanza - aprendizaje esencialmente tradicionales, la contribución de la Universidad al desarrollo de competencias se focaliza en los conocimientos y la capacidad de análisis y aprendizaje. Igualmente los 
estudios universitarios constituyen una aportación significativa en cuanto a las capacidades de trabajar en equipo y hacerse entender. Sin embargo, son estas competencias, y no las anteriores, las requeridas para el desempeño de las tareas de los trabajos que ocupan los egresados. Por el contrario, los egresados latinoamericanos experimentan cierta insuficiencia en su formación universitaria para realizar una gestión eficaz de su tiempo y afrontar adecuadamente el trabajo bajo presión. Existe una correspondencia muy ajustada entre los valores laborales de los egresados latinoamericanos y su consecución en sus puestos de trabajo. La estabilidad laboral y la posibilidad de aprender cosas nuevas, son las características que más valoran los egresados en sus trabajos. En cambio el prestigio social y el nivel de ingresos quedan relegados a las últimas posiciones en su escala de valores.

\section{Conclusiones}

Las conclusiones se presentan por cada una de las tres facultades:

Facultad de Estudios Sociales y Empresariales

- En relación con la situación sociodemográfica de los graduados de la Facultad de Estudios Sociales y Empresariales, la mayoría de los graduados son mujeres, solteras y sin hijos, sus padres tienen nivel de formación universitario, y sus madres nivel de estudios de bachillerato.

- La mayor proporción de los graduados terminaron su bachillerato entre los 15 y 18 años.

- La proporción de graduados satisfechos con las competencias adquiridas en el proceso de formación es alta en los cinco años del estudio, la proporción es de ocho satisfechos por cada 10 graduados.

- El promedio de satisfacción con las características del personal docente está por encima del $70 \%$ en todas las características, excepto con el trabajo de campo.

- El promedio de satisfacción con el apoyo a los estudiantes es diferente en cada programa al interior de la facultad.
- En todos los programas, en el factor gestión administrativa, la variable atención al personal es calificada como satisfechos entre un $61 \%$ y un $81 \%$.

- El promedio más bajo de satisfacción de los graduados con los recursos físicos se presenta con los espacios deportivos y los espacios para realizar actividades artísticas.

\section{Facultad de Ingeniería}

- En su mayoría, los graduados son solteros y no tienen hijos.

- Los padres de los graduados de esta facultad, en un porcentaje superior al $27.5 \%$ tienen un nivel de estudios profesional y con relación a las madres los porcentajes son superiores al 50\%.

- El $68 \%$ de los graduados volverían a cursar un estudio de pregrado en la institución y la principal razón para querer volver es por la calidad de la formación. De los graduados que no volverían a cursar un programa de pregrado, la principal razón que manifestaron es que el valor del programa supera disponibilidad de los recursos.

- Los graduados se encuentran en general satisfechos con los recursos ofrecidos en cuanto a personal docente, con excepción del trabajo de campo y pruebas experimentales, en donde la satisfacción es menor. El nivel de satisfacción en cuanto a apoyo a los estudiantes es mayor en los recursos de asistencia médica y psicológica y gestión de prácticas empresariales, en general el nivel de satisfacción con el apoyo a los estudiantes es menor que lo que señala estar con el personal docente.

- Los graduados de esta facultad están satisfechos con la atención del personal administrativo en porcentajes superiores al $69 \%$ y con la agilidad de los trámites administrativos los porcentajes oscilan entre el 54 y el $82 \%$ de satisfacción.

- En cuanto a los recursos físicos el nivel de satisfacción más alto es la biblioteca con un promedio de $78 \%$ y el nivel de satisfacción 
más bajo es los laboratorios y talleres con un promedio del $42 \%$.

- Los graduados tienen un nivel de satisfacción superior al $70 \%$ con las diferentes competencias adquiridas en la institución.

- En su mayoría, desean realizar estudios de especialización, seguido por maestría.

\section{Facultad de Salud}

- Los graduados, en su mayoría son del sexo femenino, solteros y no tienen hijos. Sus padres tienen nivel de formación profesional en proporciones que varían a través del periodo de estudio.

- Los graduados de esta facultad están satisfechos con el desarrollo de competencias en una relación de 8 satisfechos por cada 10 graduados, a excepción de las competencias de "identificar y utilizar símbolos para comunicarse, herramientas informáticas básicas y "ser creativo e innovador, exponer ideas por medios escritos y persuadir y convencer a sus interlocutores.

- El promedio de satisfacción de los graduados con las características del personal docente está por encima del $77 \%$.

- El promedio de satisfacción con la atención del personal administrativo es del $83 \%$ y con la agilidad para los trámites administrativos es del $75 \%$.

- El promedio más bajo de satisfacción de los graduados con los recursos físicos se presenta con los espacios deportivos y los espacios para realizar actividades artísticas.

\section{Referencias bibliográficas}

Acevedo, S., \& Gómez, S. (2015). On the proaability of access to highereducation colombia: determinants and evolution universidad de Eafit. Retrieved from www.banred.gov.co/ cites/default/files/eventos/archivos/Prob_ educacion:O.pdf de Becerra, G. M. A., González, F. A. M., Reyes, J. E. A., Camargo, F. J. S., \& Alfonso, Á. R. O. (2008). Seguimiento a egresados. Su importancia para las instituciones de educación superior. Teoría y praxis investigativa, 3(2), 61-65

Aneca, a. N. de E. de la C. y A. (2008). Informe estudiantes titulados universitador y mercado laboral. Proyecto REFLEX, 62. Retrieved from www.aneca.es

Ascun \& Red SEIS. (2006). Política para el fomento de la calidad de la educación y el compromiso social a través de los egresados. Retrieved from http://www.javeriana.edu.co/puj/oracle/egre sados/docs_red/docs/

Brunal, R., \& Martínez, C. (2008). Sitema integral de seguimiento a graduados de la Universidad de Córdoba 2007 - 2008. Montería. Disponible en: http://web.www3.unicordoba.edu.co/sites/ default/files/resultadoseguimiento2008.pdf

Corleto, A., Cooper, J., Loredo, H., \& Bolleto, L. (2006). Presencia de mujeres y hombres en la UNAM: una radiografía. México: Colección Equidad.

Jaramillo, A., \& Ruiz, I. C. (2001). Perfil socieconómico del estudiantado de EAFIT, 3(5), 140. Retrieved from http://www.eafit.edu. co/institucional/calidad-eafit/investigacion/ Documents/Perfil socioeconómico del estudiantado de EAFIT-2001.pdf

Javeriana, U. (2012). Estudio de seguimiento a recién egresados de programas académicos de pregrado de la sede central 2012 (Vol. 1). Colección análisis institucional 1. Retrieved from http://puj-portal.javeriana.edu.co/portal/ page/portal/PORTAL_VERSION_2009_2010/ resources_v4/SPEGRE2012V1.pdf

Marí Klose, P., Marí Klose, M., Granados, F. J., Granell, C. G., \& Martnez, Á. (2009). Informe de inclusión social en España 2009 (p. 297). España. 
Maya Guerra, J. I., \& Herrera Herrera, M. E. (2012). Aproximación al estado del arte sobre los estudios de desempeño de graduados de programas de educación superior. Revista Virtual Universidad Católica del Norte, 1(36), 127-157.

Ortega, L. M. M., López, E. N., Arboleda, G. I., \& Montoya, M. L. (2012). Perfil socio-laboral de los profesionales en Administración en Salud de la Universidad de Antioquia 1999-2008. Facultad Nacional de Salud Pública: El escenario para la salud pública desde la ciencia, 30(2), 9.

Men. (2012). Resultados de las condiciones laborales de los graduados de educación superior. Bogota. Recuperado de: http:// www.graduadoscolombia.edu.co/html/1732/ articles-334303_documento_tecnico_2013.pdf.

Men- Ministerio de Educación Nacional. (2008). Análisis de determinantes de la deserción en la educación superior colombiana con base en el SPADIES. Bogotá, Colombia. Retrieved from http://puj-portal.javeriana.edu.co/portal/ page/portal/PORTAL_VERSION_2009_2010/ resources_v4/Estado_del_Arte_de_la_ Retencion_de_Estudiantes.pdf.
Montenegro, I. A. (2011). Proceso de seguimiento a graduados de la Universidad del Magdalena. Retrieved from http://egresados.unimagdalena. edu.co.

OLE- Observatorio Laboral de la Educación. (2015). Observatorio Laboral de la educación. Retrieved from http://www.graduadoscolombia.edu.co/ html/1732/channel.html.

Rodriguez, P., Ramirez, R., Hincapié, J. A., Echeverri, U. C., Agudelo, A. M., Trejos, A. M., \& Patiño, H. (2012). Observatorio de graduados egresados Andina (p. 132). Ogea.

UAO- Oficina de Planeación y desarrollo Institucional. (2007). Perfil socioeconómico del estudiante autónomo, 130. Retrieved from http://www.Uao.Edu.Co/sites/default/files/ perfil_socioeco_estudiantes_0.Pdf

Unal. (2012). Ingeniería y ciencias es tema también de mujeres. Retrieved February 16, 2015, from http://noticias.universia.net.co/ vida-universitaria/noticia/2012/11/13/981835/ ingenieria-ciencias-es-tema-mujeres.html 